\title{
Laser Assited Doping for Photovoltaic Applications
}

\author{
Vikram V. Iyengar and Mool C. Gupta \\ Charles L. Brown Department of Electrical and Computer Engineering, \\ University of Virginia, Charlottesville, Va-22904 \\ E-mail:mgupta@virginia.edu
}

\begin{abstract}
We successfully demonstrate, incorporation of phosphorus and boron in silicon using an IR semiconductor diode laser $(\lambda=940 \mathrm{~nm})$ in ambient air. Commercially available transparent spin on dopants were used to obtain surface concentrations higher than $5 \times 10^{19}$ atoms $/ \mathrm{cm}^{3}$ with junction depths ranging from $20 \mathrm{~nm}$ to 3 microns at laser operating powers of 25-39 Watts. Sheet resistances values of $\sim 5$ ohms / sq were observed for both $p$ type and $n$ type doping. It was also observed that the crystalline nature of the silicon was preserved and the surface remained smooth.

DOI: 10.2961/jlmn.2009.02.0003
\end{abstract}

Keywords: Laser doping, solar cell, silicon

\section{Introduction}

The performance of c-silicon solar cells largely depends on the concentration and junction depth of the emitter region. The blue response of the solar cell and the formation of a good ohmic contact depend on the doping concentration of emitter region. Similarly, the presence of a back surface field is very important to increase the collection efficiency of the solar cell. The back surface field (BSF) basically opposes the motion of electrons to the base where the recombination velocity is very high thus improving carrier collection. The presence of a BSF also translates into higher fill factors primarily due to the smaller current induced potential drops [1].

The principle of laser doping, by surface melting and solid state diffusion, has been previously demonstrated using nanosecond lasers such as Excimer laser and $\mathrm{CW}$ $\mathrm{CO}_{2}$ laser [2-4]. The use of expensive optics for frequency doubling, beam shaping etc. hinders the possible integration of these laser systems into the manufacturing environment.

In this work we demonstrate the principle of laser doping using a semiconductor diode laser with high wall plug efficiencies ( $>50 \%$ ), excellent beam quality without the need for beam shaping and low operating powers. The use of spin on dopants (SOD) as the dopant source circumvents the use of toxic gases, time consuming and expensive surface cleaning steps and vacuum equipment.

In this paper we explore a $\mathrm{CW}$ laser based process in ambient air for the formation of emitters and back surface fields for solar cells. Another important aspect of this work is that the process has been carried out without the use of any optical absorption layers in spite of the low absorbance in silicon at $\lambda=940 \mathrm{~nm}$. Optical absorbing layers like diamond like films (DLC) at this wavelength have previously found to be very efficient for laser annealing of ion im- planted samples and laser re-crystallization [5-6]. The presence of such a carbon film could lead to unintentional incorporation of Carbon into the emitter. The use of such optical absorption layers will also increase the process complexity and cost of solar cells.

\section{Experiment}

Owing to $\mathrm{CW}$ nature of the laser used and the low absorption coefficient of silicon at $\lambda=940 \mathrm{~nm}$, doping profiles and sheet resistance needs to be investigated. At higher powers, the continuous nature of the radiation may provide enough energy for the activation of slip planes in the lattice, eventually leading to the cracking of the wafer. In order to ease the thermal stress and increase the absorption of the laser radiation, we have used a chopper with a frequency of $41 \mathrm{~Hz}$ and a substrate heater. Phenomenon like optical generation of free carriers by inter sub-band transitions result in the increase of the absorption co-efficient and could possibly aide the formation of shallow doping profiles using laser radiation with energy at the band edge of silicon. The experimental setup used is illustrated in figure 1(a). Figure 1 (b) shows the scanning pattern of the sample under the laser beam. The chopped laser beam $(41 \mathrm{~Hz}$; duty cycle: $50 \%$ ) was focused using a lens with focal length of $50 \mathrm{~mm}$. The diameter of the circular focused beam used was around 500 microns. 


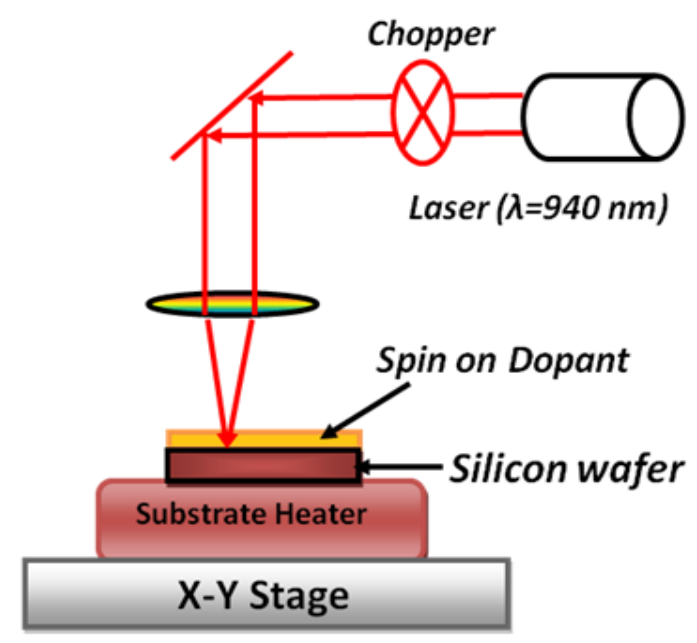

Fig.1 (a) Schematic of experimental setup; the $1 \mathrm{~cm}^{2}$ sample is placed on a heater at a constant temperature of $300^{\circ} \mathrm{C}$. The heater with the sample is mounted on a micro precision computer controlled $\mathrm{x}-\mathrm{y}$ stage.
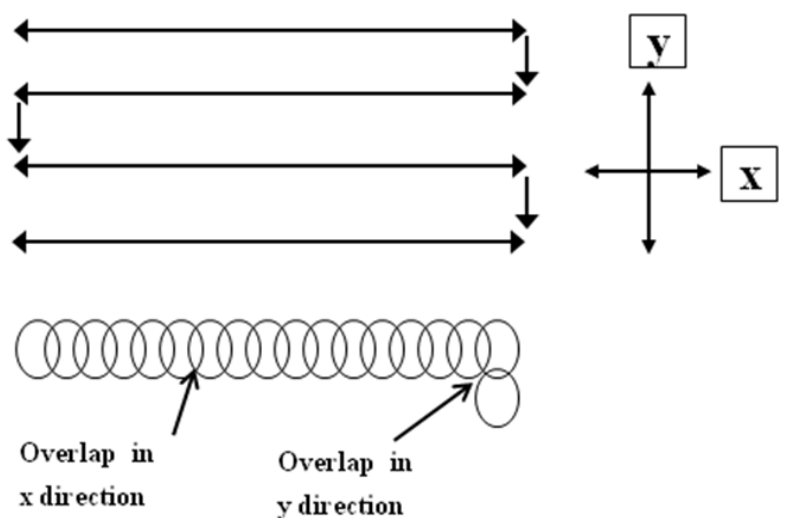

Fig.1 (b) top shows the raster scanning pattern and the bottom shows the overlap of the laser beam in the $\mathrm{x}$ and $\mathrm{y}$ direction as indicated.

We have used boron doped $\mathrm{Cz}-\mathrm{Si}$ wafers with a resistivity of 6-8 ohm-cm. The wafers were diced into smaller samples measuring $1 \mathrm{~cm}^{2}$. The samples were then cleaned using acetone, methanol and then rinsed in de-ionized water (DI) to remove any organic contaminations. The native oxide on the samples was not stripped in order to increase the adhesion of the SOD to the silicon sample. The samples were then spin coated with phosphorus or boron doped spin on dopants obtained from Emulsitone Inc. Table 1 summarizes the type of dopant, dopant concentration in SOD, spin speeds and the thickness of the final film.

Table 1 SOD Parameter Summary

\begin{tabular}{cccc}
\hline Material & Concentration & RPM & Thickness \\
\hline P-SOD & $0.03 \mathrm{M}$ & 3000 & $200 \mathrm{~nm}$ \\
B-SOD & $0.039 \mathrm{M}$ & 2500 & $500 \mathrm{~nm}$
\end{tabular}

After spin coating, the samples were first soft baked at $120{ }^{\circ} \mathrm{C}$ for 10 minutes and then hard baked at $270{ }^{\circ} \mathrm{C}$ for 10 minutes to drive away any organic contamination. Optical absorption spectroscopy of the SOD spin coated on glass slides revealed no absorption in the wavelength range of 200-1000 nm. The samples were mounted on a computer controlled $x-y$ translation stage and were scanned under the laser beam. The laser treated samples were then cleaned in a $10 \% \mathrm{HF}$ solution to strip the residual dopant layers and any oxide layer. The samples were then rinsed in DI water before any characterization.

\section{Results and Discussion}

In this section, various results on n-type and p-type laser doped samples will be presented. In all cases, the samples were held at a constant substrate temperature of $300^{\circ} \mathrm{C}$ unless mentioned otherwise. Laser doping was carried out as a function of laser power for different number of scans and sample scanning speeds. The sample was raster scanned under a laser beam using a computer controlled $\mathrm{x}$ $y$ stage with an overlap of $20 \%$ in the y direction for all cases to avoid any edge effects. The overlap in the $\mathrm{x}$ direction was varied from $42 \%$ to $80 \%$. The number of scans denotes the number of times the laser beam scans across the sample in the $\mathrm{x}$ direction (back and forth) before travelling down in the $y$ direction to repeat the process as indicated in figure 1(b). 1x, 2x, 4x, 10x scans denotes that the laser beam traveled $1,2,4$, and 10 times respectively. It was also observed that the rastering of the laser beam over a Si sample for all the processing parameters without any SOD did not result in any change in the sheet resistance. The surface morphology without the use of SOD was similar to when SOD was used.

\subsection{N-type Doping}

Results on sheet resistance of phosphorus doped samples as a function of laser power for various processing parameters will be presented in this section.

Figure 2 illustrates the sheet resistance as a function of laser power for different number of scans and chopping of the CW laser beam. It should be noted that the starting sheet resistance of the wafer is $\sim 180 \mathrm{ohms} / \mathrm{sq}$. It can be observed that at laser power less than 30 Watts for the case of the chopped laser beam, the sheet resistance of the sample is much above $180 \mathrm{ohms} / \mathrm{sq}$. This can be attributed to the compensation type of doping that first occurs on account of the starting wafer being $p$ type or the junction depth being extremely shallow. It can also be seen that the sheet resistance for the samples treated with the chopped laser beam decreases from around $400 \mathrm{ohms} / \mathrm{sq}$ to 2 ohms/sq. In the case of the laser doping achieved without 
the use of the chopper, the threshold power to achieve low sheet resistance was clearly reduced.

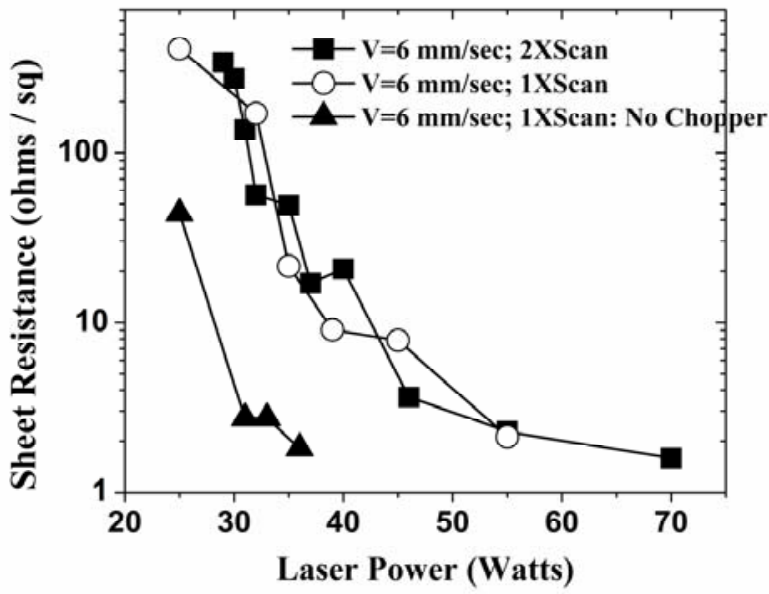

Fig. 2 Illustrates the sheet resistance of the $\mathrm{n}$ doped samples as a function of laser power for different number of scans.

This reduction in maximum was accompanied by complete surface re-melt at powers greater than 39 watts. After the removal of the SOD, it was observed that the silicon surface morphology changes and becomes translucent. XRD analysis conducted on such a surface nevertheless revealed that the crystalline nature of the material was intact.

Figure 3 (a) shows the effect of laser power on sheet resistance for different sample scanning velocities. It can be seen that the decreasing trend of sheet resistance is identical for all the stage velocities. We think that the minor difference in the sheet resistance at a particular laser power is for different sample scanning velocities is due to the change in junction depths. The stage velocity controls the laser dwelling time and hence the incident energy. The pulse energy incident in this study ranges from $261 \mathrm{~J} / \mathrm{cm}^{2}$ to $847 \mathrm{~J} / \mathrm{cm}^{2}$. This in turn can be used to control the surface temperature and time for the dopants to diffuse into the surface.

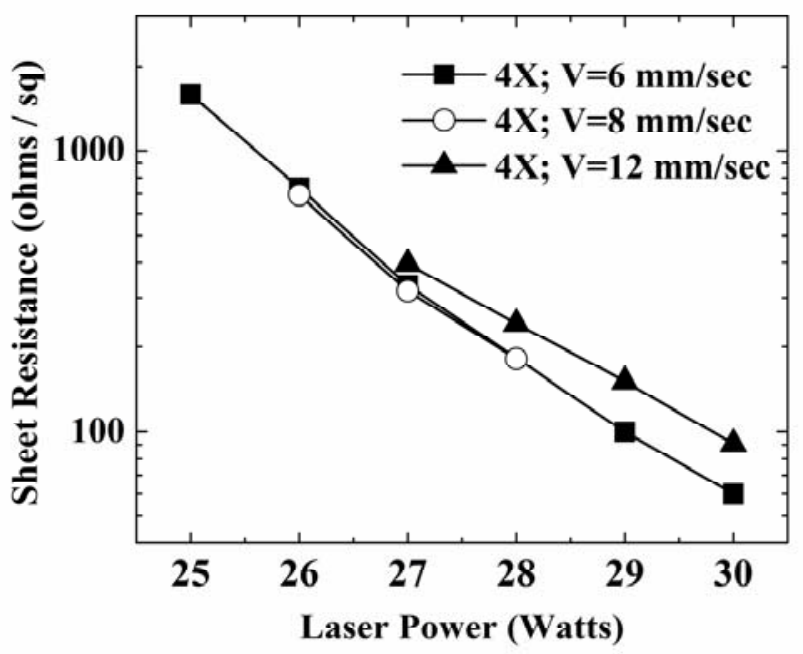

Fig.3 (a) Sheet resistance as a function of laser power for different scan velocities; number of scans was kept constant at four.

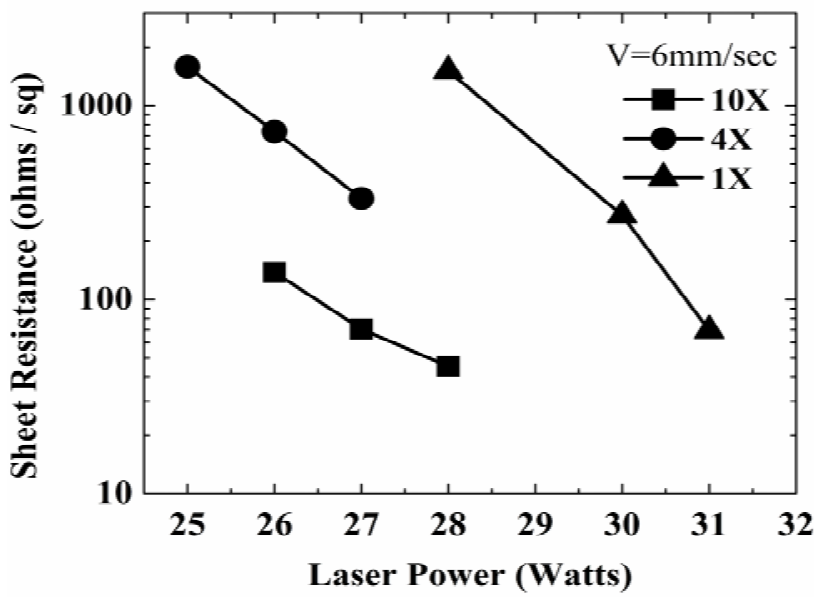

Fig. 3 (b) Effect of laser power on sheet resistance for different number of scans.

We also think that the minority carrier lifetime of the samples for $4 \mathrm{X}$ type should be less than that of $1 \mathrm{X}$ type scan owing to the change in material structure due to the increase in number of possible defect sites. Minor surface roughness and thermal stress marks were observed on the surface which arises due to the scanning nature of the laser beam. Figure 3 (b) illustrates the effect of laser power on sheet resistance for different number of scans. The sample scanning velocity was kept constant at $6 \mathrm{~mm} / \mathrm{sec}$. A small discrepancy in the measured sheet resistance for the $\mathrm{v}=6$ $\mathrm{mm} / \mathrm{sec} ; 1 \mathrm{X}$ case is observed. This can be attributed to small change in the thickness of the SOD layer which can cause a big change in the reflection and hence the absorbed energy.

It can be observed that minimum sheet resistance shifts towards lower power levels as the number of scans increase. This trend is as expected. As the number of scans increases at a constant sample scanning velocity, the sample is subjected to more exposure causing more dopant atoms to be incorporated and hence the junction depth to increase. 


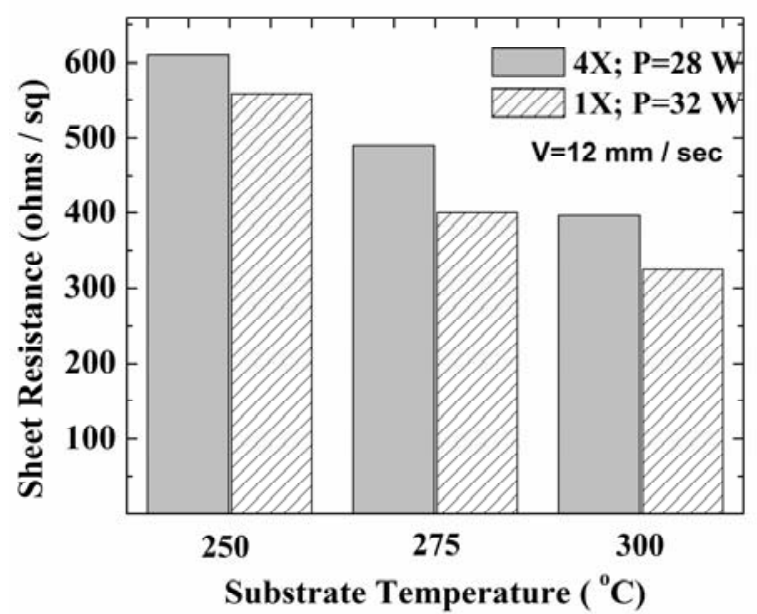

Fig.4 Effect of substrate temperature on sheet resistance for different types of scans at a constant power and scanning velocity

Figure 4 depicts the effect of initial substrate temperature on the sheet resistance. It is clear from the figure that the sheet resistance decreases as the substrate temperature increases. The trend for two different types of scans was seen to be the same. This can be explained by the increase in the absorption coefficient and hence an increase in the surface temperature. The increase in the absorption coefficient can result from phonon assisted indirect transitions across the bandgap [7] and free carrier absorption as the intrinsic carrier concentration in silicon increases with temperature [8].

Variation in concentration profile with depth was examined using Secondary Ion Mass Spectrometry (SIMS). SIMS results for two different scans and laser power is shown in figure 5 .

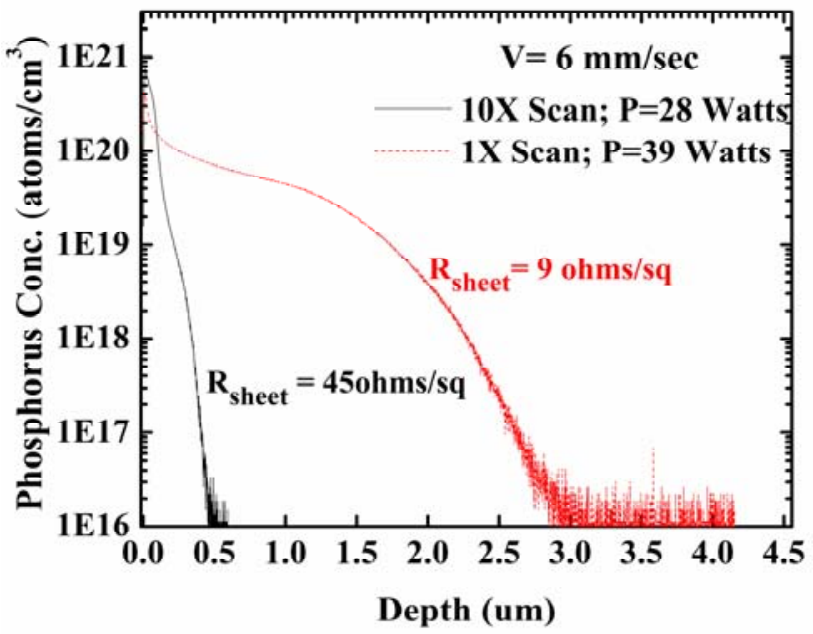

Fig. 5 SIMS box like depth profiles of phosphorus doped samples for different scan types

It can be clearly seen that by choosing the right laser power and type of scan, the doping profile can be engineered to a great extent. It can be seen that the junction depth is 0.43 and 2.5 microns for $10 \mathrm{X}$ and $1 \mathrm{X}$ type of scan respectively. Another important observation from the figure is the significant change in the surface concentration; hence by choosing the appropriate processing conditions it is possible to tailor the surface concentration which has a significant impact on the quantum efficiency in the blue region of the spectrum.

Further, it was seen that for $1 \mathrm{X}$ scan type, the junction depth was reduced to $100 \mathrm{~nm}$ by reducing the operating power of the laser to 30 Watts. It was also noted that $1 \mathrm{X}$ scan type was more preferable considering the lesser amount of time taken to scan the given area provided; secondary effects like material quality are preserved as compared to multiple scan types. Ability to tailor the junction depths precisely to such small depths also allows the use of this laser process for selective emitter fabrication [9].

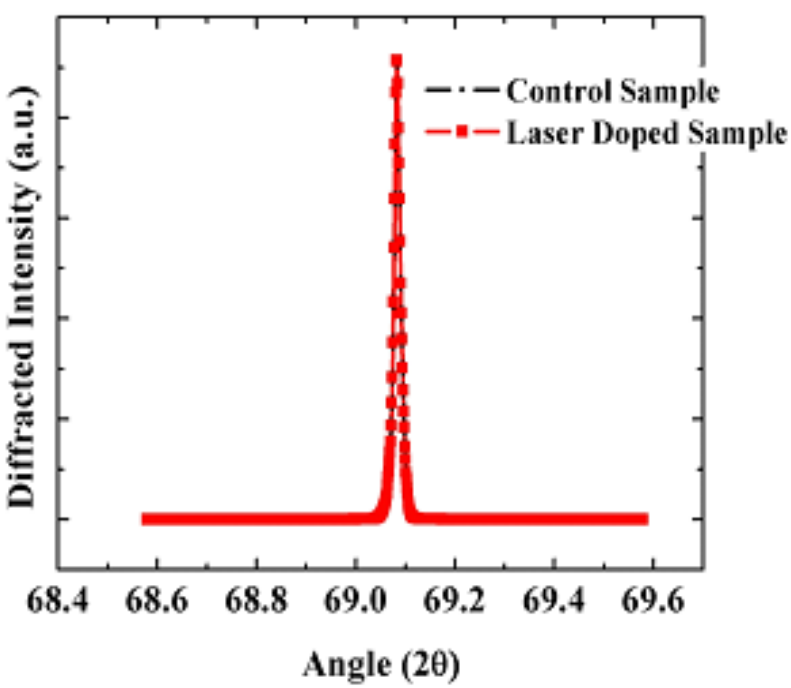

Fig. 6 XRD spectra of control and $n$ type laser doped sample

Figure 6 shows the XRD spectra of a control and laser doped sample with a junction depth of 3 microns. It can be seen that there is no apparent change in the crystalline quality after the laser doping process. Also, the presence of any amorphous silicon if any on the surface was not observed in the background as the samples were thoroughly cleaned in aqueous HF before carrying out the XRD analysis.

Figure 7 illustrates the control of dopant incorporation by laser power. 


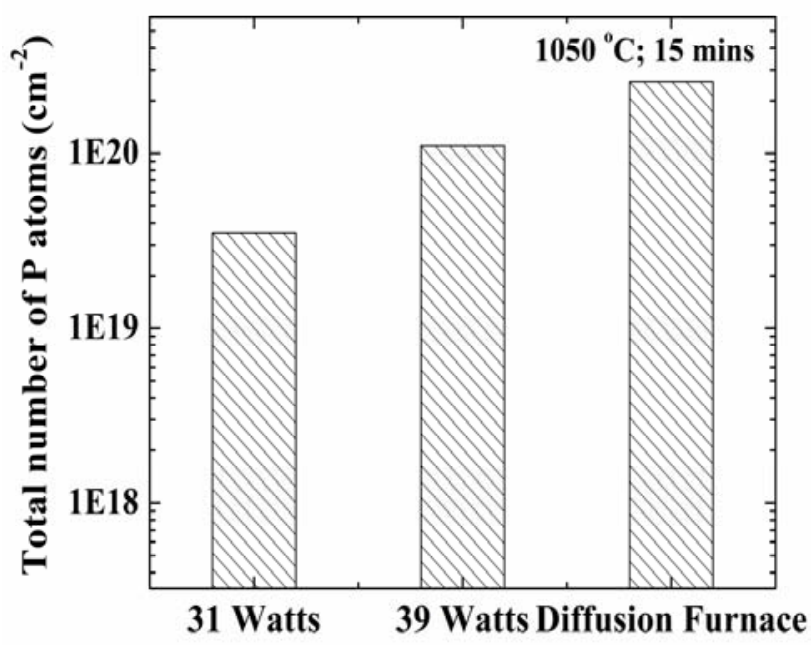

Fig. 7 Total number of phosphorus atoms incorporated as a function of laser power

The total number of incorporated phosphorus atoms is calculated by integrating the area under the doping profile obtained by SIMS. Results from phosphorus doping obtained by furnace diffusion at $1050{ }^{0} \mathrm{C}$ for 15 minutes has also been included to bench mark the results. The thickness of the SOD used for furnace diffusion was the same as that for laser doping.

Optical microscope images of laser treated samples are presented in figure 8 . Figure 8 (a) shows light re-melt marks on the sample at low operating powers; 8 (b) shows thermally induced surface roughness at higher powers.

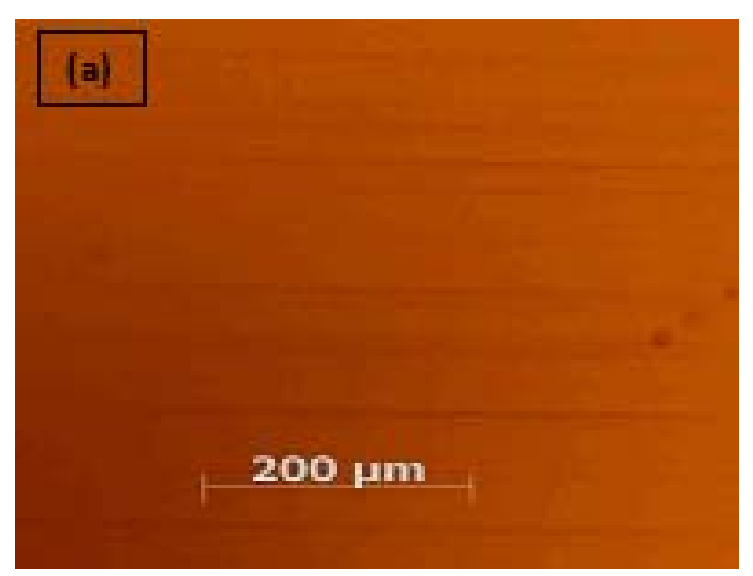

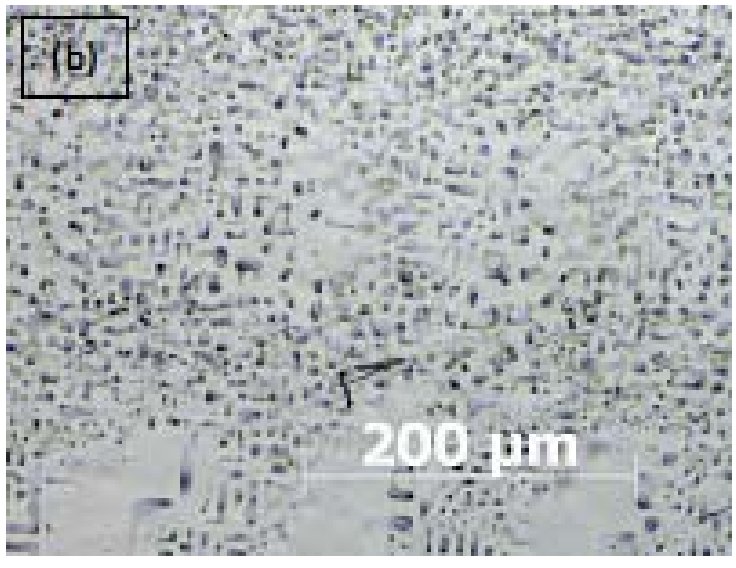

Fig. 8 Optical micrographs as a function of incident laser power; (a) power $=28$ Watts $($ b) power $=32 \mathrm{Watts}$

\subsection{P-type Doping}

$\mathrm{P}$ doped samples starting with a sheet resistance of about $180 \mathrm{ohms} / \mathrm{sq}$ were spun coat to form a p+ layer. Figure 9 illustrates the sheet resistance as a function of laser power for different scan types and scanning velocities.

It can be observed from figure 9 that the onset or threshold power of doping for $1 \mathrm{X}$ and $4 \mathrm{X}$ type scan for a scanning velocity of $12 \mathrm{~mm} / \mathrm{sec}$ is more than for $6 \mathrm{~mm} / \mathrm{sec}$ as expected. It can also be seen that the trend for $\mathrm{X}$ and $4 \mathrm{X}$ type scan at constant velocity is the same and that there is no large difference in the sheet resistance between the two. This can be explained by the deeper junction produced by the $4 \mathrm{X}$ scan type due to multiple scans.

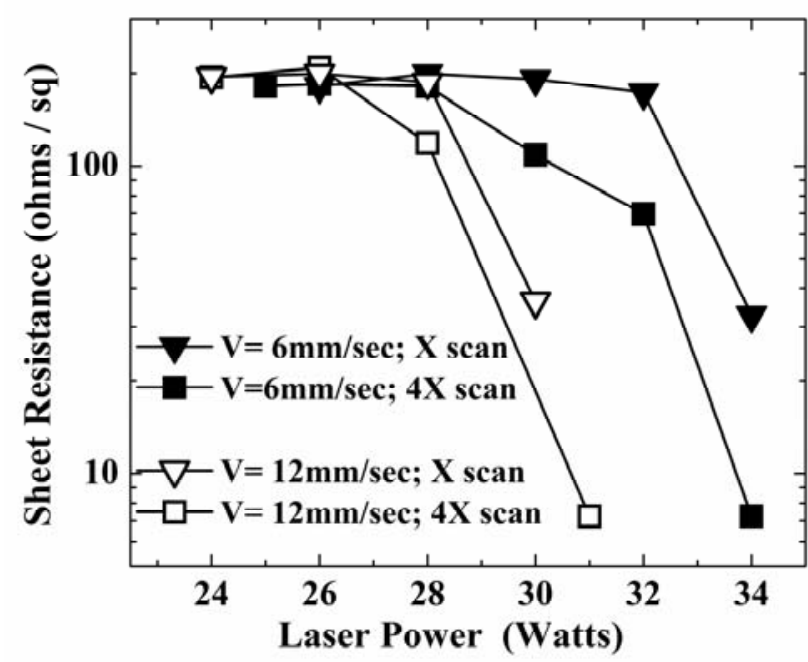

Fig. 9 Variation of sheet resistance with laser power of a $p+$ doped samples; the solid and open symbols represent $\mathrm{X}$ and $4 \mathrm{X}$ scan type for different scanning velocities. The log scale is used in order to highlight the onset of boron doping $\left(\mathrm{p}^{+}\right)$. 
Finally, figure 10 illustrates the shallow $\mathrm{p}+$ boron junction profiles obtained by SIMS. It can be clearly seen that the junction depth can be increased by the use of multiple number of scans.

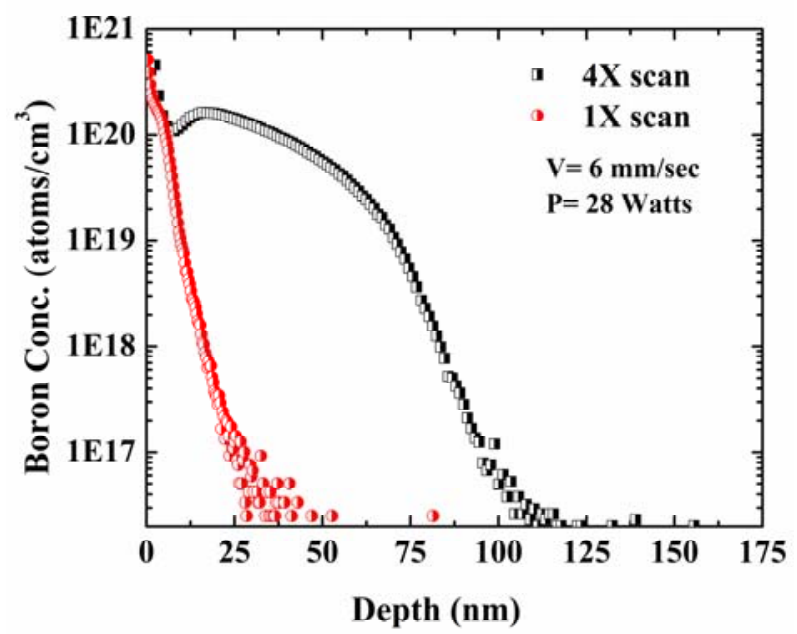

Fig. 10 SIMS depth profiles of boron doped samples for different number of scans at a constant sample scanning velocity and power.

\section{Conclusions and Future Work}

In summary, we have demonstrated the use of a highly efficient diode laser in the near infrared regime to obtain $p$ and $\mathrm{n}$ type doping. Good uniformity and profile control have been demonstrated for both boron and phosphorus incorporation into c-Si substrates. Effect of initial substrate heating to increase the absorption coefficient and reduce the thermal stress was shown and that it was not very severe. It was observed that increasing the scanning velocity raised the laser power threshold for doping of both $\mathrm{p}$ and $\mathrm{n}$ type. Finally, optical images revealed minor roughness formation and thermal re-melt lines on account of the scanning nature of the beam and it was shown this roughness in no way affected the crystalline nature of the silicon sample. These laser induced defects will be a cause of carrier recombination which can degrade the performance of solar cells. These defects could be removed by etching away few tens of nanometers of the silicon surface which is a common practice for these applications. Removal of this surface layer will also etch away any 'dead layer' that could degrade device performance.

In the future, we plan out to carry a detailed investigation of minority carrier lifetime to arrive at optimum laser processing parameters for efficient photovoltaic device fabrication.

\section{Acknowledgement}

This work was supported by the NASA Langley Research Professor Program and National Science Foundation
I/UCRC Laser Applications Center (Grant \# IIP-0537883). The author would like to thank Chen-Nan Sun for help with the laser setup. The author also wishes to thank Dr. Barada K. Nayak for useful discussions.

\section{References}

[1] J Fossum, IEEE Trans. on Electron Devices, Vol. ED . 24, No. 4, April 1977.

[2] A. M. Prokhorov, A. Yu. Bonchik, S. G. Kiyak, A. A. Manenkov, G. N. Mikhailova, A. V. Pokhmurskaja and A. S. Seferov, I. Ursu, V. Craciun and I. N. Mihailescu: Applied Surface Science, Volume 43, Issues 1-4, 2 December 1989, Pages 340-345

[3] V C Loy, Y W Wongy, H C Choy, Y Q Chenyz, S M Hoy, P W Chany and K Y Tongx, Semicond. Sci. Technol. 11 (1996) 1285-1290

[4] Caroline Carlsson, A. Esturo-Bretón, M. Ametowobla, J. R. Köhler and J. H. Werner, $21^{\text {st }}$ European Photovoltaic Solar Energy Conference, 4-8 September 2006

[5] Naoki Sano, Nobuyuki Andoh, Toshiyuki Sameshima, Yasuhiro Matsuda, and Yasunori Andoh, Japanese Journal of Applied Physics, Vol. 46, No. 25, 2007, pp. L620-L622

[6] Naoki Sano, Masato Maki, Nobuyuki Andoh, and Toshiyuki Sameshima, Japanese Journal of Applied Physics, Vol. 46, No. 3B, 2007, pp. 1254-1257

[7] H.A. Weakien and D.Redfield,J.Appl. Phys. 50(3), March 1979

[8] Sze, S. M., Physics of Semiconductor Devices, John Wiley and Sons, N.Y., 1981

[9] C. Carlson et. al., Proc. of $21^{\text {st }}$ European Photovoltaic Solar energy conference, Sept 2006, pp. 938-940

(Received: July 10, 2008, Accepted: May 1, 2009) 